\title{
Anticrossing of Spin-Split Subbands in Quasi-One-Dimensional Wires
}

\author{
A. C. Graham, M. Y. Simmons, ${ }^{*}$ D. A. Ritchie, and M. Pepper \\ Cavendish Laboratory, J. J. Thomson Avenue, Cambridge, CB3 OHE, United Kingdom
}

(Received 26 February 2008; published 6 June 2008)

\begin{abstract}
In quantum Hall systems, both anticrossings and magnetic phase transitions can occur when oppositespin Landau levels coincide. Our results indicate that both processes are also possible in quasi-1D quantum wires in an in-plane $B$ field, $B_{\|}$. Crossings of opposite-spin 1D subbands resemble magnetic phase transitions at zero dc source-drain bias, but display anticrossings at high dc bias. Our data also imply that the well-known 0.7 structure may evolve into a spin-hybridized state in finite dc bias.
\end{abstract}

The varied and complex physics of the quantum Hall ferromagnet - $\mathrm{a} 2 \mathrm{D}$ electron or hole system tuned to bring two Landau levels into coincidence - has been extensively studied both theoretically [1,2] and experimentally [3,4], and depends delicately on interaction strength, carrier density, the forms of the wave functions and spins of the coincident levels. In contrast, the magnetic properties of quasi-1D systems in the vicinity of crossings of spin-split 1D subbands [5-9] are still poorly understood, as is the case with much of the interesting physics associated with these strongly interacting quasi-1D systems.

In this Letter, we present experimental evidence that 1D subbands of opposite spin can undergo both magnetic phase transitions and anticrossings, depending on whether or not the subbands coincide near the Fermi energy $E_{F}$. It has previously been shown that crossings of Zeeman-split subbands in high $B_{\|}$exhibit nonquantized conductance structures, known as analogs, which have the same temperature $B_{\|}$and dc bias dependences as the 0.7 structure $[5,10]$. We demonstrate that although the conductance features of the crossings at zero dc bias imply an abrupt change in the magnetic polarization of the quantum wire, the finite-dc-bias features continuously evolve from one spin type to the other with increasing $B_{\|}$, while maintaining a finite energy gap, which is more reminiscent of a hybridized state or anticrossing, than an abrupt magnetic phase transition. In a finite dc bias, the lower (drain) chemical potential $\mu_{d}$ can provide information about a subband after it has populated and is well below the upper (source) chemical potential $\mu_{s}$. This allows us to study the coincidence of subbands which are heavily populated and far below $\mu_{s}$, whereas in zero dc bias, we can only study a coincidence of subbands if it occurs at $E_{F}$. We argue that this explains the difference in observed behavior in the low and high-bias regimes.

We begin by presenting quantum wire conductance data which exhibit crossings of spin-split subbands as a function of $B_{\|}$. This is to aid interpretation of the more complex finite dc bias conductance data which we go on to present, taken at five $B_{\|}$through the crossing region. The zero-bias $B_{\|}$data exhibit an anomalous discontinuity in the crossing region; nevertheless, we will show that at all fields the features can be unambiguously labeled with one spin type. In contrast, although the features in the finite-dcbias data remain clearly defined at all $B_{\|}$, we demonstrate that there is a field range in which these features cannot be labeled with a particular spin type, but are inherently ambiguous in spin character. We conclude by discussing mechanisms that could be responsible for this surprising spin effect, and possible implications for the origin of the 0.7 structure [11] and analogs [5,10].

Our samples comprise split-gate devices on a $\mathrm{GaAs} / \mathrm{Al}_{0.33} \mathrm{Ga}_{1-0.33} \mathrm{As}$ heterostructure. Samples used in this Letter have a length $0.4 \mu \mathrm{m}$ and width $0.6 \mu \mathrm{m}$, but we observe the same effects with other sample dimensions. The $292 \mathrm{~nm}$ deep two-dimensional electron gas has a mobility of $1.1 \times 10^{6} \mathrm{~cm}^{2} / \mathrm{V} \mathrm{s}$ and a carrier density of $1.15 \times 10^{11} \mathrm{~cm}^{-2}$. $B_{\|}$was applied perpendicular to the current direction, but we observe the same features in a parallel field. Hall measurements indicate the sample alignment was better than $0.5^{\circ}$. The measurement temperature was $100 \mathrm{mK}$.

Applying an in-plane $B$ field causes 1D electric subbands to Zeeman split. This is apparent in a gray-scale plot of the derivative of the differential conductance with respect to split-gate-voltage (Fig. 1). Left-moving (rightmoving) dark lines correspond to the population of lower-energy spin-down subbands (higher-energy spin-up subbands). Also visible at $\sim 9 \mathrm{~T}$ are the first crossings of opposite-spin subbands (from left to right, $1 \uparrow$ with $2 \downarrow, 2 \uparrow$ with $3 \downarrow$, etc.), and second crossings at 12-14 T (from left to right, $1 \uparrow$ with $3 \downarrow, 2 \uparrow$ with $4 \downarrow$, etc.).

Going from higher to lower subband indices (from right to left) the crossings display increasingly pronounced discontinuities, indicating that opposite-spin subbands abruptly rearrange as they populate. As we have previously noted [9], this is reminiscent of the magnetic phase transitions predicted to occur for opposite-spin Landau levels. Apart from these discontinuities, however, the crossings of the lower subbands broadly resemble those of higher subbands. Since the general features of Fig. 1 do not differ greatly from a noninteracting picture, we can assign 


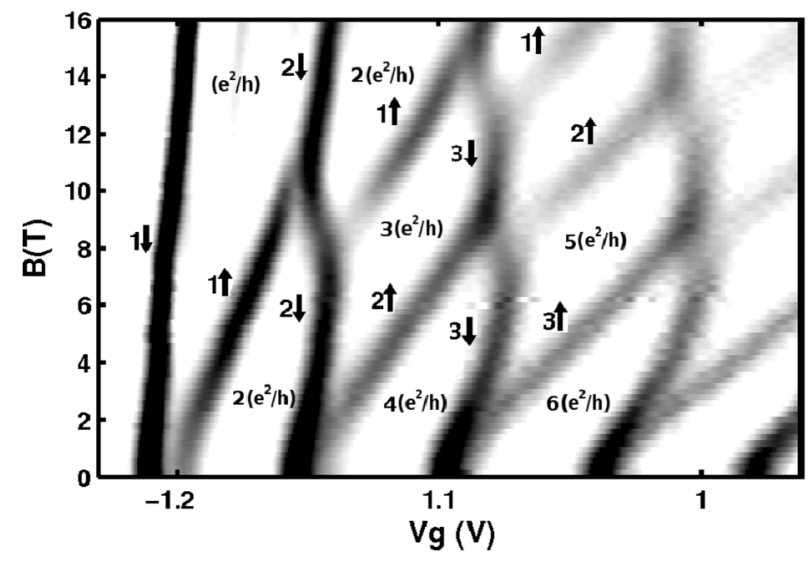

FIG. 1. Gray scale of the derivative of the differential conductance, as a function of gate voltage, for $B_{\|}=0-16 \mathrm{~T}$. Dark lines correspond to populating a spin-split 1D subband. White regions correspond to conductance plateaux - features are labeled accordingly.

spin types to the features accordingly; we have replotted the data in Fig. 2(a) and superimposed red or gray lines (blue or dark gray lines) on features associated with higherenergy spin-up (lower-energy spin-down) subbands. This is to aid identification of features in the finite-dc-bias data in Fig. 2 which are the focus of our Letter.

We now demonstrate that although there is no apparent ambiguity in the spin types of features at zero dc bias, in finite dc bias two branchlike features continuously evolve from one spin type to the other with increasing $B_{\|}$. These two finite-bias features do not display any obvious discontinuity as a function of $B_{\|}$, or any other indication that a change of spin type has occurred - it is only by following the features at zero dc bias that this change in spin type becomes apparent. At $B_{\|}=5 \mathrm{~T}$, the data broadly resemble a noninteracting picture. As illustrated in Fig. 2(b), each dark point at zero dc bias, corresponding to a sharp increase in differential conductance due to the populating of a 1D subband, splits into a pair of $V$-shaped dark branches with increasing dc bias; the left (right) branch corresponds to the subband intercepting the higher-energy source chemical potential $\mu_{s}$ (lower-energy drain chemical potential $\mu_{d}$ ). We therefore assume that the spin type of a branch in finite dc bias will be the same as the spin type of the feature at zero dc bias that the branch has split from [see Fig. 2(b)]. Thus, since Fig. 2(a) shows that the secondand third-from-left features at $5 \mathrm{~T}$ are spin up ( $1 \uparrow$ subband) and spin down ( $2 \downarrow$ subband), respectively, we have labeled the right-moving branch $\boldsymbol{A}$ second from left in Fig. 2(c), $B=5 \mathrm{~T}$, as spin up, and the right-moving branch $\boldsymbol{B}$ third from left as spin down.

We will follow the evolution of branches $\boldsymbol{A}$ and $\boldsymbol{B}$ in particular as $B_{\|}$is increased and subbands $1 \uparrow$ and $2 \downarrow$ cross. From 5 to $6.6 \mathrm{~T}$, the branches have only moved slightly, and the spin types of the features are not yet ambiguous. Although, again, by $7.8 \mathrm{~T}$, branches $\boldsymbol{A}$ and $\boldsymbol{B}$ have slightly

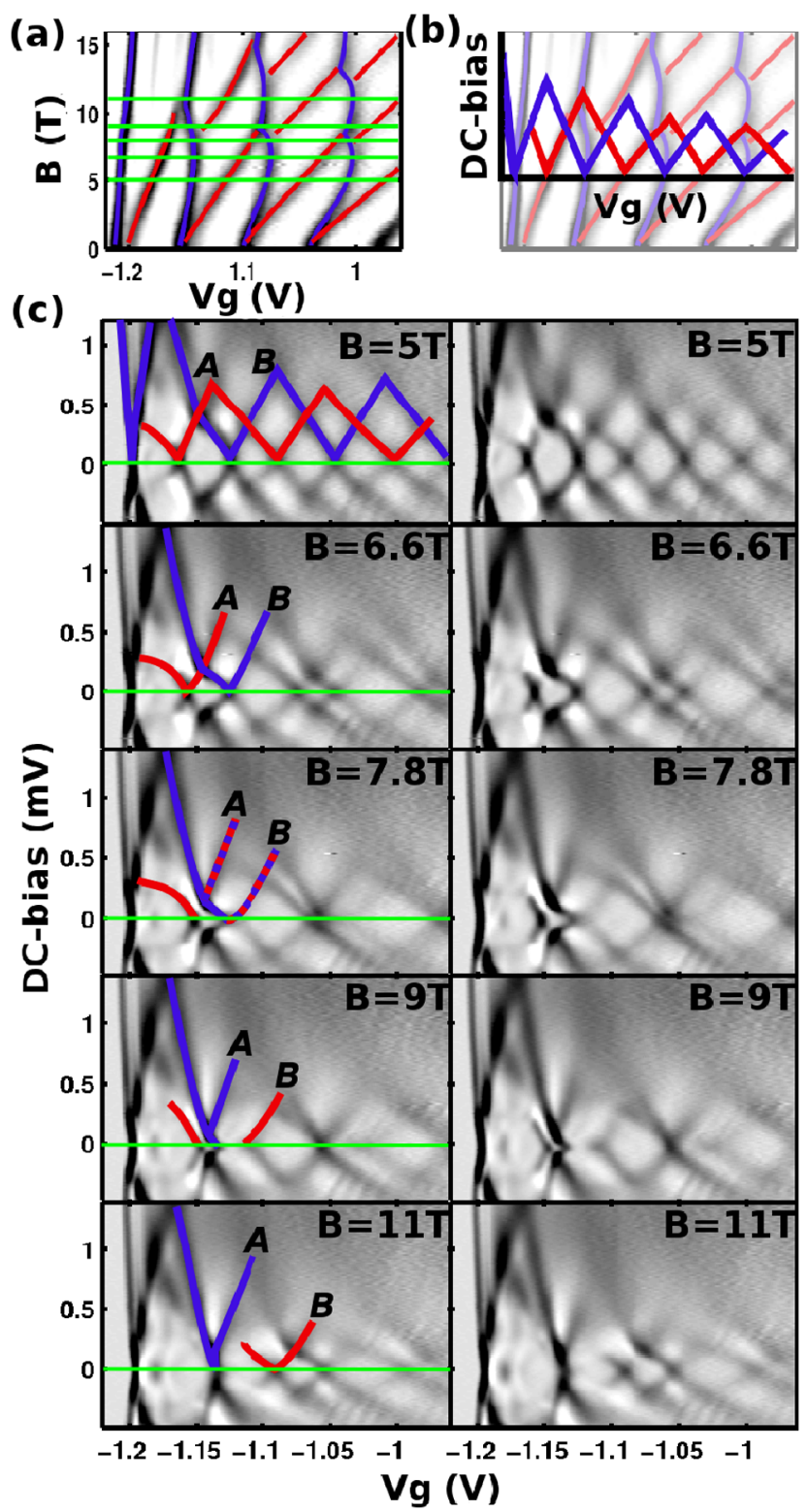

FIG. 2 (color online). dc bias spectroscopy in the crossing region of $1 \uparrow$ and $2 \downarrow$. Lower-energy spin-down features are marked blue or dark gray, and higher-energy spin-up features are marked red or gray. (a) Gray-scale plot, as in Fig. 1, showing the evolution of quantum wire conductance characteristics in an in-plane $B$ field. Green or light gray lines indicate the fields at which dc bias data in (c) were taken. (b) Schematic illustrating how we have assigned spin types to features in the dc bias data-at a fixed $B_{\|}$, we assume that a spin-up (spin-down) feature at zero dc bias splits into two spin-up (spin-down) features in finite-bias. (c) Gray scale of the derivative of the differential conductance, as a function of gate voltage, for dc biases from -0.5 to $1.4 \mathrm{mV}$, at $B_{\|}$marked with green or light gray lines in (a) - the spin types of the features along a green or light gray line in (a) enable us to identify the spin types of the finite-bias features. Data on the left are reproduced without the annotation on the right for clarity. The spin types at $B=7.8 \mathrm{~T}$ are ambiguous, however, and imply that an anticrossing is occurring. 
changed position compared to $6.6 \mathrm{~T}$, the data still look broadly similar. Note, however, that although these features at zero bias have moved closer together by $7.8 \mathrm{~T}$ because we are approaching the crossing of $1 \uparrow$ and $2 \downarrow$, branches $\boldsymbol{A}$ and $\boldsymbol{B}$ in finite dc bias are almost as far apart in gate voltage as they were at $5 \mathrm{~T}$. Assigning the spins associated with these branches at $7.8 \mathrm{~T}$ does not appear problematic, as they have only moved slightly from $6.6 \mathrm{~T}$. However, the dc bias data taken at $9 \mathrm{~T}$, which is in the crossing region, reveals a dramatic complication. At zero dc bias, branch $\boldsymbol{B}$ has split apart from the left branch it was joined to at $7.8 \mathrm{~T}$, and now has no accompanying left branch, the significance of which was discussed in Ref. [10]; furthermore, the feature at zero dc bias that branch $\boldsymbol{B}$ evolves from is now spin up instead of spin down, according to Fig. 2(a) at 9 T. Therefore, although the form of branch $\boldsymbol{B}$ changes very little from 5 to $9 \mathrm{~T}$, at $5 \mathrm{~T}$ it is associated with the $2 \downarrow$ subband, but at $9 \mathrm{~T}$ it is associated with the $1 \uparrow$ subband. Thus, assigning spin types to the features at $7.8 \mathrm{~T}$ is not actually straightforward-they are ambiguous so are marked with both colors.

By $11 \mathrm{~T}$, the dc bias data once again broadly resemble a noninteracting picture - each feature at zero dc bias splits into two branches at finite dc bias, and there is no apparent difficulty in assigning a subband and spin index to the branches. At this field it is now clear that branch $\boldsymbol{A}$ must be associated with the $2 \downarrow$ subband instead of the $1 \uparrow$ subband, because it splits from a spin-down feature at zero dc bias, so it too has changed its spin type between 5 and $11 \mathrm{~T}$. At higher fields (not shown), branch $\boldsymbol{A}$ remains unchanged, but branch $\boldsymbol{B}$ takes part in a second crossing, this time with $3 \downarrow$, and reverses its spin again. In summary, at zero dc bias [Figs. 1 and 2(a)], features of opposite spin move very close to each other in gate voltage as $B_{\|}$increases, and for lower subband indices, give rise to a crossing with an abrupt discontinuity that resembles a magnetic phase transition; however, in finite dc bias [Fig. 2(c)], although features $\boldsymbol{A}$ and $\boldsymbol{B}$ of opposite spin maintain a large gap in gate voltage between them at all $B_{\|}$, they appear to evolve continuously into the opposite spin type as they pass through the crossing region-there is no discontinuity in the gate-voltage position of branches $\boldsymbol{A}$ and $\boldsymbol{B}$ with increasing $B_{\|}$. This is more reminiscent of an anticrossing than a phase transition.

Both magnetic phase transitions and anticrossings between opposite-spin Landau levels can occur in quantum Hall systems and have now been thoroughly characterized, theoretically and experimentally. In contrast, crossings between opposite-spin 1D subbands in quantum wires are poorly understood, and as yet, no rigorous theoretical framework exists to describe them. Hence, we cannot definitively explain the nature of these 1D subband crossings in zero and finite bias at present. However, our data imply that magnetic phase transitions and anticrossings can both occur in quantum wires, with magnetic phase transitions occurring for zero or low-dc bias, and anticrossings in the high-dc bias regime. We will proceed by identifying some differences and similarities between 1D-subband crossings and Landau-level crossings, and then use these to discuss why anticrossings between 1D subbands of opposite spin in a quantum wire might be observed specifically in the finite-bias regime.

Whereas a Landau level contains a finite number of states which are filled completely as it passes below $E_{F}$, a 1D subband has states which extend to infinite energies - no matter how far below $E_{F}$ the subband edge is, there will still be states in that subband available at $E_{F}$. On the one hand, if $E_{F}$ in a quantum Hall system is set at $\nu=2$ filling factor, the system can remain completely unpolarized with equal numbers of spin-up and spin-down electrons in $n=0 \downarrow$ and $n=0 \uparrow$, until a magnetic phase transition occurs, which will then give a completely polarized system with only spin-down electrons in $n=0 \downarrow$ and $n=1 \downarrow$. In a $1 \mathrm{D}$ system, however, as $B_{\|}$is increased from zero towards the crossing of the $1 \uparrow$ and $2 \downarrow$ subbands, the system becomes increasingly polarized, because $1 \uparrow$ continuously depopulates while $2 \downarrow$ increasingly populates. For this reason alone, it is no surprise that a 1D-subbandcrossing is qualitatively different to a Landau-levelcrossing.

A second key difference between the two systems is that density and confining potential are set to some fixed values in a quantum Hall system and opposite-spin Landau levels are made to cross by varying $B_{\|}$alone, using different tilt angles. In quantum wires, however, both density and confining potential are varied along with $B_{\|}$in order to induce crossings. Given that the nature of Landau-level crossings delicately depends on density and confinement, this is likely to be true of $1 \mathrm{D}$ subband crossings also-it is possible that the character of the subband crossing changes as the gate voltage is swept through the crossing region.

Additionally, the finite-bias regime in a quantum wire has no analogy in a quantum Hall system. So far, there has yet to be any theoretical study of this regime in a realistic interacting quasi-1D electron system. It is, however, becoming increasingly apparent that dc bias spectroscopy provides powerful insight into electron interactions in quantum wires. This is because in a finite bias, the lower (drain) chemical potential provides information about a subband even after it has populated and is well below the upper (source) chemical potential.

We suggest that the key difference between the crossings we observe in the zero- and finite-bias regimes is this: at zero dc bias, both subbands taking part in the crossing are very close to $E_{F}$, and therefore do not have a large number of occupied states, whereas at a "right branch" in finite dc bias, both of the subbands are near the drain chemical potential, and therefore have many occupied states. In the case of coincident Landau levels, the nature 
of the crossing greatly depends on whether one, both, or neither of the levels are occupied, and whether they are near $E_{F}$. If both or neither are occupied and they are not near $E_{F}$ when they cross, then an anticrossing is likely. However, if they are near $E_{F}$, and only one is occupied, then a paramagnetic-ferromagnetic phase transition becomes possible, with all the electrons abruptly emptying from, for example, the $n=0 \uparrow$ spin-up level, and filling the $n=1 \downarrow$ Landau level instead, to create a completely spinpolarized system. There is no energy saving to be made by the levels rearranging in this way if both are well below $E_{F}$ or well above it when they coincide - in this situation, the levels hybridize instead and anticross. Therefore, in the case of 1D subbands near the drain chemical potential at large dc bias, they are so far below the source chemical potential when they coincide that a phase transition is no longer possible. However, in the presence of exchange interactions or spin-orbit coupling, mixing of spins can occur, and the levels will instead anticross.

The large enhancement of spin splitting which allows crossings to be observed at around $10 \mathrm{~T}$ instead of around $50 \mathrm{~T}$ for bare Zeeman splitting, is good evidence that exchange interactions in quantum wires are strong, and aided by the electron-electron interaction could mix the opposite-spin subbands. Additionally, the numerous differences which have been observed in the conductance characteristics of spin-up and spin-down subbands in finite $B_{\|}$ $[10,12,13]$ constitutes further evidence of strong exchange interactions.

It is less clear whether spin-orbit coupling will play a role here. Although the Dresselhaus contribution to spinorbit coupling for electrons in GaAs (due to crystal inversion asymmetry) is usually negligibly small, typical Rashba contributions to spin-orbit coupling (due to electric fields associated with confining potentials) are considerably larger [14]. Since quantum wires are electrostatically confined in two directions, it has been argued that Rashba spin-orbit coupling is particularly important in these systems [15]. Irrespective of the strength of spin-orbit coupling, the electron interaction coupled with exchange could mix spins and give the observed anticrossing between 1D subbands of opposite spins.

Lastly, we note that the crossing region displays all of the same characteristics as the lowest plateau in zero $B_{\|}-$ they both exhibit nonquantized conductance structures, the 0.7 structure and analogs, which evolve into quantized structures with increasing $B_{\|}$. These structures weaken and rise in conductance with decreasing temperature, and strengthen and rise in conductance under finite bias. Furthermore, the dc bias gray scale at $B=9 \mathrm{~T}$ in Fig. 2(c) shows that the right-branch labeled $\boldsymbol{B}$ has no accompanying left branch, unlike the data at lower and higher $B_{\|}$; such anomalous behavior also typifies the dc bias dependence of the 0.7 structure at zero $B_{\|}$, as was discussed in Ref. [10]. This similarity of the 0.7 structure to the crossing region implies that it may also evolve into a spin-hybridized state under a finite dc bias-clearly further investigation is required, in order to establish whether this is indeed the case.

In conclusion, we have presented experimental evidence that $1 \mathrm{D}$ subbands of opposite spin may be able to hybridize, creating an anticrossing when they are tuned to coincide in a large in-plane $B$ field. We only observe anticrossings in high dc biases, however. At zero-to-low dc bias, subbands of opposite spin appear to abruptly rearrange, giving a discontinuity when they cross; this does not resemble an anticrossing, but rather, a magnetic phase transition. We suggest that the difference between the low and high dc bias regime is that at low dc biases, we can only observe subbands coinciding if this occurs at $E_{F}$ - this happens to be the regime in which a magnetic phase transition is possible. At high dc biases, however, the lower-energy drain chemical potential allows us to study the coincidence of subbands which have populated at much higher energies. Since magnetic phase transitions [16] are only expected to occur for subbands near $E_{F}$, subbands which coincide near the drain chemical potential in high dc biases may instead anticross, as is implied by our data. This result emphasizes the wealth of interesting physics which can occur in quasi-1D quantum wires.

We acknowledge useful discussions with C.H.W. Barnes, C. J. B. Ford, M. Kataoka, F. Sfigakis, T.-M. Chen, J.P. Griffiths, K.-F. Berggren, and B. Spivak. This work was supported by EPSRC, U.K. A.C. G. acknowledges support from Emmanuel College, Cambridge.

*Present address: University of New South Wales, School of Physics, Sydney, NSW 2052, Australia.

[1] G.F. Giuliani and J. J. Quinn, Phys. Rev. B 31, 6228 (1985).

[2] T. Jungwirth and A.H. MacDonald, Phys. Rev. B 63, 035305 (2000).

[3] W. Desrat et al., Phys. Rev. B 71, 153314 (2005).

[4] A. J. Daneshvar et al., Phys. Rev. Lett. 79, 4449 (1997).

[5] A. C. Graham et al., Phys. Rev. Lett. 91, 136404 (2003).

[6] A. J. Daneshvar et al., Phys. Rev. B 55, R13 409 (1997).

[7] G. Grabecki et al., Physica (Amsterdam) 13E, 649 (2002).

[8] K.-F. Berggren, P. Jaksch, and I. Yakimenko, Phys. Rev. B 71, 115303 (2005).

[9] A.C. Graham et al., Solid State Commun. 131, 591 (2004).

[10] A. C. Graham et al., Phys. Rev. B 75, 035331 (2007).

[11] K. J. Thomas et al., Phys. Rev. Lett. 77, 135 (1996).

[12] A. C. Graham et al., Phys. Rev. B 72, 193305 (2005).

[13] A.C. Graham et al., Physica (Amsterdam) 22E, 264 (2004).

[14] T. Hassenkam et al., Phys. Rev. B 55, 9298 (1997).

[15] A. V. Moroz, K. V. Samokhin, and C. H. W. Barnes, Phys. Rev. Lett. 84, 4164 (2000).

[16] S. M. Girvin, Phys. Today 53, No. 6, 39 (2000). 